\title{
Engineering the plasmon resonance of large area bi-metallic nanoparticle films by laser nanostructuring for chemical sensors
}

\author{
Michail J. Beliatis, Simon J. Henley*, S. Ravi P. Silva \\ Nano-Electronics Centre, Advanced Technology Institute, University of Surrey, Guildford, GU2 7XH, United Kingdom. \\ *Corresponding author: s.henley@surrey.ac.uk
}

\begin{abstract}
Large area fabrication of metal alloy nanoparticles with tunable surface plasmon resonances, on low cost substrates is reported. A UV excimer laser was used to anneal 5nm thick Ag Au bilayer films deposited with different composition ratios to create alloy nanoparticles. These engineered surfaces are used to investigate how the wavelength of the surface plasmon resonance affects the optical detection capability of chemical species by Surface Enhanced Raman Spectroscopy.
\end{abstract}

Metal nanoparticle films (MNFs) are attracting considerable attention in the fields of Raman biosensors[1, 2], gas sensors[3], solar cells[4, 5] and light emitting diodes[6] due to enhancement in light coupling efficiency between the surface plasmons (SP) developed at the nanoparticles and the device. Use of metal alloy nanoparticles can optimize the surface effect of enhanced Raman scattering (SERS) by tuning their plasmon resonance absorption close to the laser frequency used to probe the Raman signal from molecules on their surface $[7,8]$. However, despite these attractive properties, challenges exist regarding large scale adoptation of plamonic nanoparticle surfaces. One of the major challenges in device fabrication is the production of uniform MNFs over large area substrates and repeatable tune-ability of their surface plasmon resonances (SPR). The maximum energy coupling in SERS coincides with the wavelength of the metal nanoparticles SPR and is localized at "hotspots" [7, 9, 10].

Changes in the SPR optical absorption due to different composition of $\mathrm{Ag}$ and $\mathrm{Au}$ in alloy nanoparticles has been demonstrated previously $[11,12]$. The effect is attributed to the change in the dielectric function of the alloy nanoparticles from the volumetric contributions of $\mathrm{Ag}$ and $\mathrm{Au}$. Unlike many other fabrication methods, which include chemical synthesis[13], pulsed laser deposition [14], or thermal annealing[15], pulsed laser nanostructuring of thin metal films can be applied over large areas at low cost to fabricate supported nanoparticle surfaces. Henley et a[16] and Trice et a[17] studied the process for single metal films. Herein, we present the synthesis and characterization of metal alloy nanoparticles with tunable SPR by laser nanostructuring of Ag Au bi-layer films and discuss how the position of the maximum absorption affects the SERS efficiencies.

Corning glass substrates where cleaned in a ultrasonication bath for $10 \mathrm{~min}$ in acetone and blow dried with nitrogen. Metal films were deposited in two layers by evaporating silver and gold separately. The total film thickness was fixed at $5 \mathrm{~nm}$ while the thickness of each individual layer was deposited according to $\left(\mathrm{Ag}_{\mathrm{x}} \mathrm{Au}_{5 \mathrm{x}}\right)$ where $\mathrm{x}=1,2,3,4,5 \mathrm{in} \mathrm{nm}$. The average deposition rate for all materials was $0.1-0.2 \AA / \mathrm{s}$ and a pressure of $1.0 \mathrm{x}$ $10^{-6}$ Torr was maintained in the evaporation chamber during this process. A Lambda-Physik LPX 210i excimer laser operating at $248 \mathrm{~nm}$, with a pulse duration of $25 \mathrm{~ns}$ was used for the rapid annealing. All the samples were mounted on $\mathrm{X}-\mathrm{Y}$ translation stage moving at a typical speed of $60 \mathrm{~mm} / \mathrm{min}$ where the whole sample (up to 6 " in diameter) could be annealed at the same fluence. A Gaussian profile pulse with $176 \mathrm{~mJ} / \mathrm{cm}^{2}$ fluence and repetition frequency of $15 \mathrm{~Hz}$ was used to nanostructure the films and create uniform coatings of alloy nanoparticles. After the rapid annealing a strong change in color was observed in the irradiated paths (see Figure 1 (a-f)) compared to the blue-grey initial color. This color change is attributed to the generation of metal nanoparticles with SPR absorption in the visible regime.

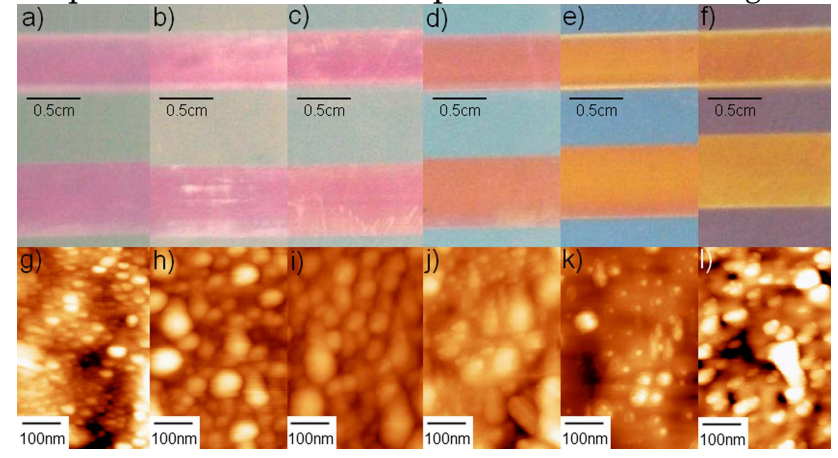

Figure 1 (Color online) (a) $\mathrm{Ag}_{0} \mathrm{Au}_{5}$ (b) $\mathrm{Ag}_{1} \mathrm{Au}_{4}$ (c) $\mathrm{Ag}_{2} \mathrm{Au}_{3}$ (d) $\mathrm{Ag}_{3} \mathrm{Au}_{2}(\mathrm{e}) \mathrm{Ag}_{4} \mathrm{Au}_{1}$ (f) $\mathrm{Ag}_{5} \mathrm{Au} 0$ alloy nanoparticles fabricated using the laser nanostucturing process and (g-l) their AFM characterization.

An Atomic Force Microscope (AFM) from Digital Instruments (Nanoscope IV, Dimension 3100) was used to map the post-annealed films as shown in Figure $1(\mathrm{~g}-1)$. Particles with average sizes in the range $2-60 \mathrm{~nm}$ were observed in all samples except from the samples $\mathrm{Ag}_{2} \mathrm{Au}_{3}$ and $\mathrm{Ag}_{3} \mathrm{Au}_{2}$ where two distinct ranges of sizes where observed 5-19nm and $20-60 \mathrm{~nm}$. 
(a)
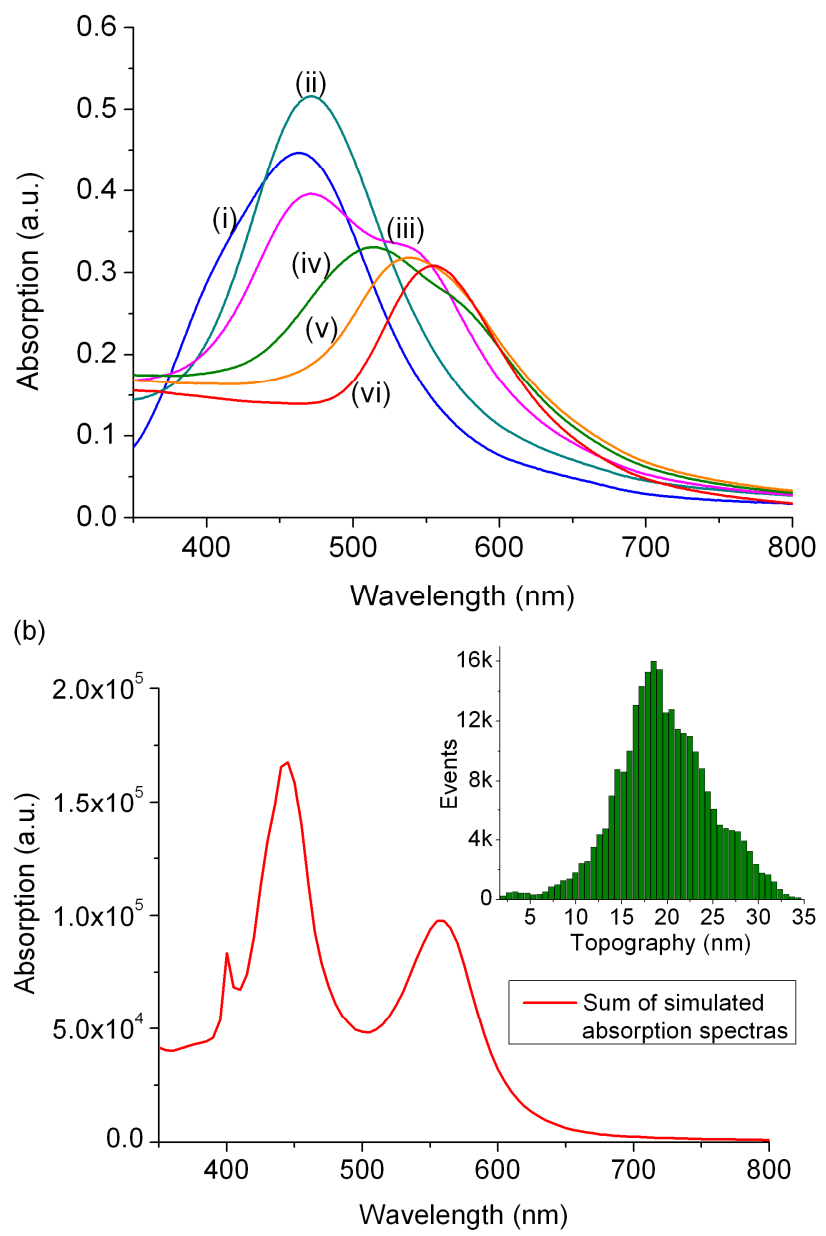

Figure 2 (Color online) (a) Optical absorption spectrum obtained from the metal nanoparticle films (i) $\mathrm{Ag}_{5} \mathrm{Au}_{0}$ (ii) $\mathrm{Ag}_{4} \mathrm{Au}_{1}$ (iii) $\mathrm{Ag}_{3} \mathrm{Au}_{2}$ (iv) $\mathrm{Ag}_{2} \mathrm{Au}_{3}$ (v) $\mathrm{Ag}_{1} \mathrm{Au}_{4}$ (vi) $\mathrm{Ag}_{0} \mathrm{Au} 5$, and (b) the statistically approximated absorption for the $\mathrm{Ag}_{3} \mathrm{Au}_{2}$ film and the $\mathrm{AFM}$ characterization is showing in the inset figure.

Figure 2 (a) shows the optical absorption of the annealed MNFs using a spectrophotometer (Varian Cary 5000). Absorptions acquired at random positions gave similar characteristics indicating uniformity over the annealed areas. A theoretical study[18] has been performed to determine the Statistical approximate Absorption (SA) for a film composed from a mixture of pure Ag and Au nanoparticles at different diameters. The simulation revealed two distinct peaks exist for such film, and therefore the single peak observed in the real measurements is attributed to alloyed nanoparticles. For the SA (Figure 2 (b)) AFM data extracted from the real films were used in the calculations with equation (1)

$$
S A(\lambda)=\sum_{d} Q \times f(d) \times \alpha_{d}(\lambda)
$$

Where $Q$ the scaling factor based on composition, $f(d)$ the frequency of events per diameter $d$ and $\alpha$ the theoretical absorption.

The surface Plasmon resonance is red shifted and broadened as the composition of $\mathrm{Ag}$ in the alloy is reduced. Our observations agree with previous studies for similar systems[11]. The exception to this is for the alloy composition with $\mathrm{x}=4$, where the intensity of the peak is higher compared with the pure Ag film. The statistical analysis combining the $\mathrm{Ag}_{3} \mathrm{Au}_{2}$ film simulation data at different size distributions (Figure 2(b)) shows that increasing the particle size affects the absorption intensity significantly, while the position of SPR absorption peak remains almost unaffected. This simulation confirms that the shift in the absorption peak observed is due to variations in the dielectric function of nanoparticles, which is caused by the different alloy compositions. Thus the bi-modal size distribution for $\mathrm{x}=2$ and 3 is not the main reason for the dual maximum in the absorption peaks. These dual absorption peaks should be attributed to composition variations. This dual peak absorption phenomenon has significant importance because it demonstrates that energy absorption can be increased over wide range of wavelengths. This is vital especially for solar cell applications where it is required to increase the absorption efficiency over the entire range of the sun's radiated wavelengths, even if the primary absorption band of the photoactive material may not be initially able to absorb the energy as a single photon process.

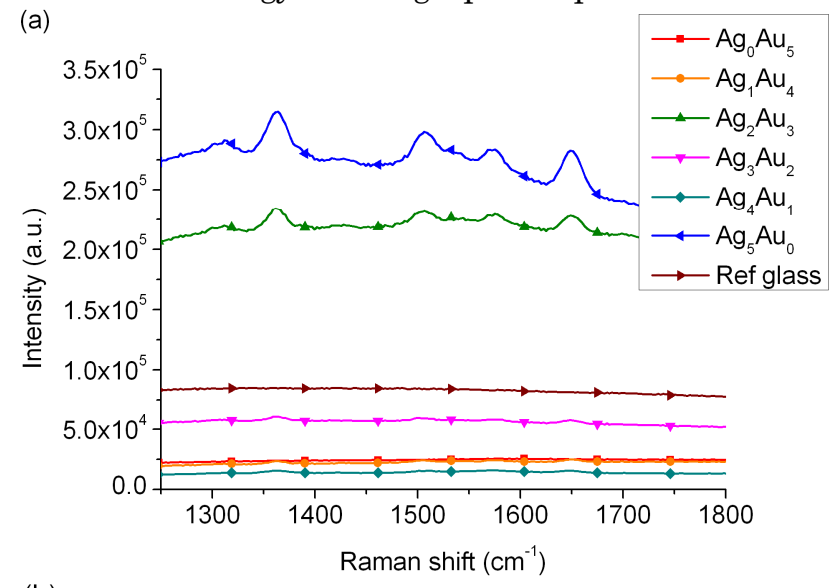

(b)

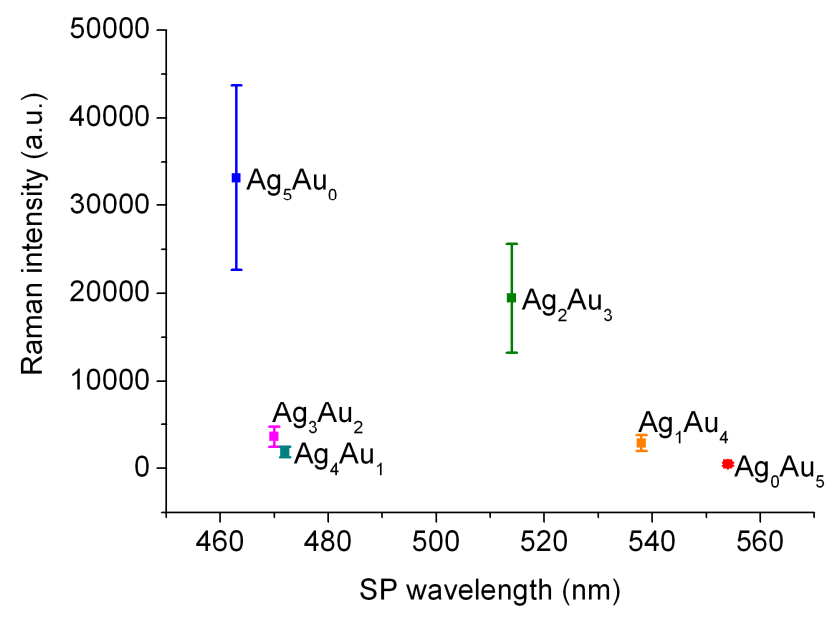

Figure 3 (Color online) (a) Typical R6G Raman signature from the MNFs samples, (b) Raman intensity versus SPR position for each alloy composition.

A $1 \mu \mathrm{M}$ aqueous solution of Rhodamine 6G (R6G) was used to investigate the SERS enhancement of the MNFs. A single $5 \mu \mathrm{L}$ droplet was placed on each substrate. All substrates were enclosed in a petri dish for $20 \mathrm{~min}$ and 
allowed to dry, controlling the rate of evaporation of water molecules to ensure uniform distribution of test molecules over the substrates. Distilled water was used to wash off the excess R6G, and the sample was blown dry with nitrogen. Raman spectra were recorded using a Renishaw micro-Raman 2000 system with a $514 \mathrm{~nm}$ laser at $4 \mathrm{~mW}$ and a $25 \%$ power attenuator. A (50X) optical lens was used to focus the laser down to an approximately $1 \mu \mathrm{m}$ diameter spot. The detector integration time was set at 1 sec and 50 accumulations at the same location were acquired to improve the signal-to-noise ratio. Figure 3 (a) shows the typical R6G Raman scattering spectra taken from the different alloy MNFs. Different enhancement factors were observed from each different alloy composition. It should be noted that no Raman signal were detected for this concentration of R6G on plain glass substrates (Ref. at Figure 3 (a)) although high fluorescence recorded. A clear Raman signal from untreated substrates could only be observed for $1 \mathrm{mmol}$ solution, a 1000x higher concentration than was deployed on SERS substrates.

The method used to apply the test analyte gave uniformly distributed films across the substrates. Therefore, the difference in the enhancement is considered to be due to different coupling efficiencies in the energy transferred from the surface plasmon resonance to the "hot spots" for the analyzed species. The intensity of the Raman scattering at the $1361 \mathrm{~cm}^{-1}$ peak versus the $\mathrm{SP}$ absorption maximum wavelength is plotted in (Figure $3(\mathrm{~b})$ ).

One can see that the Raman intensity for the $\mathrm{Ag}_{2} \mathrm{Au}_{3}$ with SPR maximum at $514 \mathrm{~nm}$ is higher than most of the other films. This confirms experimentally that SERS is higher for SPR wavelengths close to the probe laser wavelength. The high Raman intensity for the plain $\mathrm{Ag}$ nanoparticles is attributed, we believe, to the strong R6G binding affinity to silver altering the concentration of R6G in that area and hence the Raman intensity. In addition, the SERS enhancement for pure Ag films is in general stronger than for pure $\mathrm{Au}$ hence the enhancement of $\mathrm{Ag}_{2} \mathrm{Au}_{3}$ is more significant.

In conclusion, a method to fabricate large area plasmonic substrates with metal alloy nanoparticles and tunable SP resonance is demonstrated. The tune-ability of the SP wavelength is shown to modify the SERS enhancement of R6G delivered to the nanostructured surface. It is suggested that the laser nanostructuring method could be applied for fabrication of a wide range of metal alloy nanoparticle films over large areas for sensing or energy coupling applications.

The authors thank EPRSC for funding support.

\section{References}

1. S. J. Henley, and S. R. P. Silva, "Laser direct write of silver nanoparticles from solution onto glass substrates for surface-enhanced Raman spectroscopy," Appl. Phys. Lett. 91, 023107 (2007)

$2 . \quad$ N. M. B. Perney, J. J. Baumberg, M. E. Zoorob, M. D. B. Charlton, S. Mahnkopf, and C. M. Netti, "Tuning localized plasmons in nanostructured substrates for surface-enhanced Raman scattering," Opt. Express 14, 847-857 (2006).

3. M. J. Beliatis, N. A. Martin, E. J. Leming, S. R. P. Silva, and S. J. Henley, "Laser Ablation Direct Writing of Metal Nanoparticles for Hydrogen and Humidity Sensors," Langmuir 27, 1241-1244 (2010).

4. H. A. Atwater, and A. Polman, "Plasmonics for improved photovoltaic devices," Nature Mater. 9, 205-213 (2010).

5. G. D. Spyropoulos, M. Stylianakis, E. Stratakis, and E. Kymakis, "Plasmonic organic photovoltaics doped with metal nanoparticles," Photonics and Nanostructures - Fundamentals and Applications, DOI: 10.1016/j.photonics.2010.1009.1001 (2010).

6. R. Marcilla, D. Mecerreyes, G. Winroth, S. Brovelli, M. d. M. R. Yebra, and F. Cacialli, "Light-emitting electrochemical cells using polymeric ionic liquid/polyfluorene blends as luminescent material," Appl. Phys. Lett. 96, 043308 (2010).

$7 . \quad$ T. W. H. Oates, and S. Noda, "Thickness-gradient dependent Raman enhancement in silver island films," Appl. Phys. Lett. 94, 053106 (2009).

8. S. Grésillon, L. Aigouy, A. C. Boccara, J. C. Rivoal, X. Quelin, C. Desmarest, P. Gadenne, V. A. Shubin, A. K. Sarychev, and V. M. Shalaev, "Experimental Observation of Localized Optical Excitations in Random Metal-Dielectric Films," Phys. Rev. Let. 82, 4520 (1999).

$9 . \quad$ C. Hrelescu, T. K. Sau, A. L. Rogach, F. Jackel, and J. Feldmann, "Single gold nanostars enhance Raman scattering," Appl. Phys. Lett. 94, 153113 (2009).

10. D. Choi, Y. Choi, S. Hong, T. Kang, and L. P. Lee, "Self-Organized Hexagonal-Nanopore SERS Array," Small 6, 1741-1744 (2010).

11. M. Gaudry, J. Lermé, E. Cottancin, M. Pellarin, J. L. Vialle, M. Broyer, B. Prével, M. Treilleux, and P. Mélinon, "Optical properties of (AuxAg1-x)n clusters embedded in alumina: Evolution with size and stoichiometry," Phys. Rev. B 64, 085407 (2001).

12. G. C. Papavassiliou, "Surface plasmons in small AuAg alloy particles," J. Phys. F: Metal Phys. 6, L103 (1976).

13. L. Rivas, S. Sanchez-Cortes, J. V. García-Ramos, and G. Morcillo, "Mixed Silver/Gold Colloids A Study of Their Formation, Morphology, and Surface-Enhanced Raman Activity," Langmuir 16, 9722-9728 (2000).

14. T. Donnelly, S. Krishnamurthy, K. Carney, N. McEvoy, and J. G. Lunney, "Pulsed laser deposition of nanoparticle films of Au," Appl. Surf. Sci. 254, 1303-1306 (2007).

15. J. D. Carey, L. L. Ong, and S. R. P. Silva, "Formation of low-temperature self-organized nanoscale nickel metal islands," Nanotechnology 14, 1223 (2003).

$16 . \quad$ S. J. Henley, J. D. Carey, and S. R. P. Silva, "Pulsedlaser-induced nanoscale island formation in thin metal-on-oxide films," Phys. Rev. B 72, 195408 (2005).

17. J. Trice, D. Thomas, C. Favazza, R. Sureshkumar, and R. Kalyanaraman, "Pulsed-laser-induced dewetting in nanoscopic metal films: Theory and experiments," Phys. Rev. B 75, 235439 (2007).

18. B. K. Juluri, J. Huang, and L. Jensen, "Extinction, Scattering and Absorption efficiencies of single and multilayer nanoparticles," (2010), pp. doi:10254/nanohub-r18228.10252. 